\title{
LA INDUSTRIA MEXICANA A PRINCIPIOS DEL SIGLO XXI: LAS CAPACIDADES TECNOLÓGICAS Y LA INVERSIÓN EXTRANJERA COMO DETERMINANTES
}

\author{
Dmitri Fujii* \\ Luis Daniel Torres** \\ (Recibido: Febrero 2011 / Aprobado: Mayo 2011)
}

\begin{abstract}
RESUMEN
Los cambios en el modelo de desarrollo y el acelerado proceso de globalización en la competencia por mercados domésticos y externos han presentado a las empresas mexicanas un reto de dimensiones considerables. A través de las actividades de ciencia, tecnología e innovación, la generación de capacidades tecnológicas juega un papel fundamental en sus posibilidades de éxito dentro de este ambiente. El presente trabajo estudia la generación de capacidades tecnológicas para conocer la estructura y desempeño de la industria manufacturera en México a principios del siglo XXI, destacando el papel de la inversión extranjera directa. Con base en estos factores se realiza una clasificación de sectores que han desarrollado capacidades tecnológicas (High-Tech) y sectores que no lo han hecho (Low-Tech). Se
\end{abstract}

* Profesor-Investigador del Colegio Mexiquense. Correo electrónico: <dmitri.fujii@gmail.com>.

** Profesor de la Facultad de Economía de la UNAM. Correo electrónico: <luis.daniel.torres@, gmail.com>.

Los autores agradecen el apoyo de Enrique Dussel en la obtención de información no disponible en el sitio web del INEGI. 
hace especial énfasis a la disponibilidad de información estadística y sus limitantes, lo cual permitirá sentar una base sobre la que se desarrollen estudios futuros. Palabras clave: capacidades tecnológicas; inversión extranjera directa, manufactura, clasificación industrial, México

Clasificación JEL: L1, L6, 03

\begin{abstract}
Changes in the development model and the accelerated process of globalization in the competition for domestic and foreign markets have presented a challenge Mexican companies of considerable size. Through activities in science, technology and innovation, technological capacity building plays a key role in your success in this environment. This paper studies the generation of technological capabilities to understand the structure and performance of manufacturing in Mexico in the early twenty-first century, emphasizing the role of foreign direct investment. Based on these factors is a classification of sectors that have developed technological capabilities (High-Tech) and sectors that have not (Low-Tech). Special emphasis is placed on the availability of statistical information and its limitations, which will provide a basis on which to developed future studies.

Keywords: capabilities, foreign direct investment, manufacturing, industrial classification, Mexico

JEL Classification: L1, L6, 03
\end{abstract}

\title{
1. INTRODUCCIÓN
}

Es bien sabido que en las últimas décadas la economía mexicana, en particular la industria manufacturera, ha sufrido grandes cambios no sólo en su estructura sino también en las políticas gubernamentales en pro de un determinado modelo de desarrollo. Después de abandonar por completo el esquema de sustitución de importaciones, en la industria mexicana se implementó un modelo de corte neo- 
FuJII OlechKo / TORReS GonZÁlez La industria mexicana a principios del siglo XXI: las capacidades tecnológicas y la inversión extranjera como determinantes

liberal que planteaba la nula intervención de las autoridades en la regulación del desempeño de los sectores productivos.

Aunado a lo anterior, a mediados de la década de los noventa se firmó el Tratado de Libre Comercio de América del Norte (TLCAN), con lo que la industria manufacturera mexicana se vio involucrada, de la noche a la mañana, en un ambiente globalizado en el que las empresas nacionales tenían que competir no sólo con las demás empresas domésticas, sino también con una gama de empresas trasnacionales que, si bien ya habían iniciado su entrada al país, se vieron favorecidas con este tratado para abarcar nichos en los mercados mexicanos que antes no hubieran tenido a su alcance.

En este nuevo ambiente de negocios las empresas mexicanas se vieron obligadas a redoblar esfuerzos para ser competitivas a nivel local y a nivel global. Los esfuerzos en ciencia, tecnología e innovación han sido cruciales para la generación de capacidades tecnológicas que fortalecen la posición de las empresas domésticas de cara a la competencia en mercados nacionales e internacionales. Una variable adicional que hay que tomar en cuenta en el esquema planteado es la inversión extranjera directa (IED) en México, cuyas cifras han ido en claro incremento desde mediados de los noventa y que han tenido impacto en el desarrollo de la industria mexicana.

Es por ello que el presente trabajo se basa en dos aspectos clave para entender la estructura y el desempeño de la industria manufacturera mexicana de principios de la presente década: la generación de capacidades tecnológicas y la IED. Son estos dos aspectos los que permiten hacer una clasificación inédita para la industria mexicana a fin de encontrar los sectores High-Tech (que han desarrollado capacidades tecnológicas en años recientes) y los sectores Low-Tech (que no han desarrollado dichas capacidades tecnológicas).

El presente trabajo sigue de cerca la metodología de otro análisis reciente (Fujii, 2004b, 2010) pero, a diferencia del anterior, incorpora el análisis de la IED como uno de los factores clave para la determinación de la estructura de las manufacturas mexicanas. Es así que, en la primera sección del trabajo se hace un análisis de la literatura más reciente sobre capacidades tecnológicas, haciendo énfasis en el papel de la IED en la generación de dichas capacidades y en el impacto sobre la industria mexicana en general para los años recientes. 
En la segunda sección se presenta la metodología utilizada en el análisis para determinar las industrias High-Tech/Low-Tech, así como el análisis de la disponibilidad de datos sobre la industria mexicana en años recientes, la cual ha representado una seria limitante para que existan múltiples análisis de todo tipo para México. En la tercera sección se presentan los resultados del ejercicio de clasificación industrial con la metodología propuesta. Por último, en la cuarta sección se presentan algunas recomendaciones de política en términos de generación de capacidades tecnológicas en la industria manufacturera mexicana y la atracción de IED hacia el país.

\section{REVISIÓN DE LITERATURA}

El objetivo de esta sección es presentar la literatura más reciente sobre generación de capacidades tecnológicas, desde su concepción hasta la aplicación para la industria manufacturera mexicana. Asimismo, se presentan los trabajos más recientes sobre la IED y su impacto en México, tanto en términos de derramas (spillovers), como en su relación con la generación de capacidades tecnológicas en las empresas mexicanas.

\section{GENERACIÓN DE CAPACIDADES TECNOLÓGICAS}

Joseph Schumpeter (1934) fue sin duda el primer economista en subrayar la importancia de la innovación y la tecnología para el éxito empresarial. Su principal argumento se basa en el monopolio como un mal necesario para el progreso económico: el poder monopólico es más propenso a estimular la innovación y el progreso tecnológico que los mercados competitivos. En la perspectiva de este autor, la protección de ideas (patentes) es crucial, ya que con esta protección se generan incentivos para que las empresas inviertan en innovación. Adicionalmente, Schumpeter divide el proceso de cambio tecnológico en tres etapas: invención (generación de nuevas ideas), innovación (desarrollo de nuevas ideas en productos y procesos vendibles) y difusión (esparcimiento de productos y procesos) (Stoneman, 1995).

Los estudios tradicionales (neoclásicos) hacen más énfasis en los problemas asociados a la transferencia de tecnología avanzada de los países desarrollados a 


$$
\begin{array}{r}
\text { FUJI OLECHKO / TORRES GONZÁLEZ } \\
\text { La industria mexicana a principios del siglo XXI: } \\
\text { las capacidades tecnológicas y la inversión extranjera como determinantes }
\end{array}
$$

los países en vías de desarrollo que a una revisión de las estructuras innovativas y productivas en los segundos. Por lo general, el manejo de tecnología avanzada en estos países se asume como pobre y se tiene la creencia de que los países en vías de desarrollo no han desarrollado habilidades o conocimiento, puesto que únicamente importan tecnología avanzada del exterior (Fransman, 1985; Lall, 1992).

Sin embargo, desde la década de los ochenta, ha habido un importante cambio en la perspectiva de estudio de la tecnología en los países en vías de desarrollo. A partir de las teorías evolucionistas de Nelson y Winter (1982), esta nueva corriente literaria hace énfasis en la adopción de nuevas tecnologías y el cambio tecnológico en los países en vías de desarrollo. Los trabajos que siguieron al estudio de Nelson y Winter han basado su análisis en el concepto de capacidades tecnológicas ${ }^{1}$ de las empresas de estos países. La creencia generalizada es que estas capacidades se van acumulando a través del tiempo y que son elementos fundamentales para determinar si una empresa es exitosa o no en la industria de un país en vías de desarrollo (Fransman, 1985; Lall, 1992; Arias, 2003).

En otras palabras, en la literatura reciente se ha hecho énfasis en que el uso y la adopción de tecnología avanzada no es un proceso inmediato pues, depende de las habilidades desarrolladas previamente por una empresa o un sector industrial (Fransman, 1985). En este sentido, se argumenta que esta habilidad y conocimiento tecnológicos no se distribuye de manera homogénea entre empresas e industrias, sino que depende de sus esfuerzos previos y el desarrollo de capacidades (Lall, 1992; Hernández y Sánchez, 2003). En este tenor, Cohen y Levinthal (1990) señalan que la experiencia de las empresas en una tarea específica puede influir y mejorar su desempeño en el aprendizaje de tareas subsecuentes. Esta experiencia es definida por los autores como "capacidad de absorción de las organizaciones". Estudios empíricos recientes (a nivel de empresa y de industria) han sugerido la existencia de una serie de actividades que tienden a incrementar las capacidades tecnológicas de las empresas. Entre otras se señalan el perfeccionamiento de la tecnología, desarrollo de nueva tecnología y capacitación laboral. Fransman (1985); Dosi, et al. (1988); Cohen y Levinthal (1990); Lall (1992); Teece, et al.

${ }^{1}$ Definidas como la habilidad de hacer un uso efectivo del conocimiento tecnológico (Arias, 2003). 
(1994); Hernández y Sánchez (2003) plantean medidas concretas para estas actividades y, con ello, la determinación del nivel de capacidades tecnológicas.

\section{LA INVERSIÓN EXTRANJERA DIRECTA: ASPECTOS TEÓRICOS}

Un aspecto fundamental que contempla la teoría de inversión extranjera es el impacto de ésta sobre la economía receptora. En relación a ello pueden distinguirse dos posturas claramente opuestas: una que sostiene que la IED es indispensable para romper los círculos viciosos de bajo ahorro y baja productividad que ocurren en los países en vías de desarrollo; y, otra que afirma que este tipo de inversión contribuye a crear situaciones de imperfección en los mercados que resultan dañinas para las empresas domésticas en este tipo de economías. ${ }^{2}$

Dentro del debate anterior es importante señalar que la inversión extranjera puede tener diferentes impactos sobre la economía receptora. Un primer impacto puede ser observado a nivel agregado (macroeconómico), pues los flujos de IED representan una entrada de divisas a la economía receptora. De esta manera, la inversión extranjera puede influir en la expansión de dicha economía dado que representa aportaciones de capital para inversiones productivas. Este efecto se mide en la balanza de pagos, donde las inversiones extranjeras a largo plazo quedan registradas en la cuenta de capital. ${ }^{3}$

Otro efecto importante de la IED se presenta a nivel de empresa pues, la entrada de nuevas tecnologías, procesos y estándares provenientes del exterior tiene un impacto directo sobre la estructura industrial receptora. Por lo general, cuando una compañía trasnacional abre una subsidiaria en otro país ésta última se beneficia del conocimiento tecnológico desarrollado por la compañía matriz. Esta tecnología avanzada representa, entonces, parte importante de sus ventajas res-

\footnotetext{
${ }^{2}$ El debate actual puede verse con mayor profundidad en Moran (1998).

${ }^{3}$ Cabe mencionar que la inversión extranjera directa puede impactar más de una vez la balanza de pagos. En primera instancia, la IED representa un ingreso neto de divisas al país, sin embargo, conforme pasa el tiempo, habrá flujos de repatriación de beneficios hacia la casa matriz de la empresa trasnacional, así como la importación de insumos para la producción o exportaciones de las subsidiarias, con lo cual se impacta nuevamente la balanza de pagos (Stern, 1973; Krugman y Obstfeld, 2000).
} 
FuJII OlechKo / ToRres GonZÁleZ La industria mexicana a principios del siglo XXI:

las capacidades tecnológicas y la inversión extranjera como determinantes

pecto a las empresas locales más familiarizadas con el mercado (Dunning, 1977; Markusen, 1996).

En este sentido, las empresas extranjeras generalmente gozan de una presencia importante y pueden influir en la estructura industrial en términos de avance tecnológico. Desde la perspectiva de la economía receptora de IED, es claro que una de las ventajas sea que estas empresas locales, en muchos casos menos competitivas que las trasnacionales, puedan adquirir parte del conocimiento tecnológico, administrativo o de manejo de mercado que aquellas poseen.

Sin embargo, todavía no está del todo claro mediante qué mecanismos es factible la trasmisión de tecnología hacia las empresas domésticas, ni cuál es el papel que juegan las trasnacionales en el proceso. Blömstrom y Kokko (1997) justifican esta incertidumbre por el hecho de que la tecnología es un concepto no tan fácilmente medible, y por que existen diversos canales de difusión tecnológica (formales e informales) en los que la empresa trasnacional puede desempeñar un papel activo o pasivo.

Siguiendo la misma línea, el efecto que tiene la IED sobre la estructura industrial puede ser de tipo directo o indirecto. El efecto directo implica que el inversionista extranjero no se apropia de todo el incremento en la eficiencia que se genera, sino que existen ganancias para los trabajadores locales (mayores salarios), consumidores locales (menores precios) y gobierno local (mayor recaudación fiscal). El efecto indirecto, por otro lado, se presenta en forma de spillovers (Blömstrom y Persson, 1983).

El razonamiento que respalda el concepto de spillovers es que las empresas domésticas resultan beneficiadas en términos de productividad por la presencia de grandes trasnacionales en sus respectivos sectores. La hipótesis detrás de este efecto es que, después de la entrada de empresas extranjeras, las domésticas se volverán más productivas, con lo que mantendrán sus ganancias y podrán competir con aquéllas. En este escenario, las empresas locales se verán forzadas a utilizar más eficientemente las tecnologías existentes y/o estudiar posibles alternativas tecnológicas (Blömstrom, 1986; Blömstrom, Kokko y Zejan, 2000).

Si bien es incierto el papel que juegan las empresas trasnacionales en el proceso de transferencia tecnológica hacia los países en vías de desarrollo, no se puede 
decir lo mismo de la actitud que debe tener la empresa receptora de tecnología. La literatura sobre capacidades tecnológicas hace énfasis en la necesidad de un rol activo dadas las incertidumbres que existen en el proceso de adopción de nuevas tecnologías (Fransman y King, 1985). En este sentido, la teoría predice experiencias contrastantes en el desarrollo tecnológico, pues el conocimiento tecnológico (acumulado a través de un proceso de aprendizaje de la empresa) es desigual entre empresas (Dosi, et al.1988; Lall, 1992). En otras palabras, se espera que el éxito en la adopción de nuevas tecnologías esté condicionado a la capacidad tecnológica de cada empresa receptora.

De acuerdo a lo anterior, la teoría sobre inversión extranjera plantea que las empresas domésticas resultarán beneficiadas de la presencia de empresas trasnacionales, las cuales, a través de nuevas tecnologías e impacto sobre sus respectivos mercados, generarán un incremento en la productividad de las locales. Estas últimas, sin embargo, no tienen un éxito tecnológico garantizado pues, más bien ello depende del desarrollo de sus propias capacidades tecnológicas.

\section{LA INVERSIÓN EXTRANJERA DIRECTA: EVIDENCIA EMPÍRICA}

A partir de la década de los setenta la literatura registra un creciente interés por determinar si existe evidencia empírica de los efectos que genera la presencia de empresas trasnacionales en el sector industrial de la economía receptora de IED. Uno de los principales objetivos de los estudios realizados ha sido verificar la presencia de posibles spillovers de productividad. Aunque los primeros análisis empíricos se enfocan a países desarrollados (Caves, 1974 y Globerman, 1979), la mayoría de los estudios posteriores se basan en datos industriales para países en vías de desarrollo. ${ }^{4}$

\footnotetext{
4 Aquí se describen únicamente los trabajos dedicados a México. Sin embargo, existe un grupo relativamente amplio de estudios sobre otras economías en desarrollo los cuales reportan resultados diversos. Algunos de estos estudios son los de Aitken y Harrison (1991, 1999) para Venezuela; Haddad y Harrison (1993) para Marruecos; Kokko, Tansini y Zejan (1996) para Uruguay; Sjöholm (1999) y Blomström y Sjöholm (1999) para Indonesia; Liu (2001, 2002, 2006) para China; Eeckhout y Jovanovic (2002) para Estados Unidos o Pavcnik (2002) para Chile.
} 
FuJII OLEChKo / TORRES GONZÁLEZ La industria mexicana a principios del siglo XXI: las capacidades tecnológicas y la inversión extranjera como determinantes

Los trabajos de Blomström y Persson (1983) y Blomström (1986) sobre la industria manufacturera mexicana pueden ser considerados como los pioneros en el área. En ambos se realiza un análisis a nivel sectorial (con 215 y 145 sectores, respectivamente) que sugiere un impacto positivo de la presencia de capital externo sobre la productividad de la industria, así como un impacto positivo de la presencia de empresas extranjeras sobre la productividad de las domésticas. Es decir, en ambos casos, se comprueba la presencia de spillovers de productividad.

Al igual que en los estudios descritos anteriormente, los de Kokko (1994) y Blomström, Kokko y Zejan (1993) utilizan datos para la industria manufacturera mexicana que reportan la presencia de spillovers. En el primero el autor analiza la relación entre spillovers y la brecha tecnológica entre empresas domésticas y extranjeras. La muestra de 216 sectores se divide en grupos de alta y baja tecnología. Los resultados revelan que los spillovers son más comunes en sectores de baja tecnología, sin embargo se argumenta que no existe evidencia de que éstos no se presenten en el otro grupo.

El segundo trabajo incluye datos de adquisición de tecnología (pagos por importación de patentes, licencias y asistencia técnica) para 144 sectores. Los autores buscan comprobar si la competencia entre empresas locales y extranjeras ha incitado a las primeras a importar tecnología. Sus resultados muestran una relación positiva entre importaciones tecnológicas de las empresas extranjeras y las de empresas domésticas que buscan competir con ellas. El efecto es más claro para bienes intermedios y de consumo.

En un enfoque distinto, Aitken, Hanson y Harrison (1997) presentan un análisis para verificar la presencia de spillovers asociados a la exportación en México. La hipótesis central del estudio es que, las actividades exportadoras de las empresas extranjeras producen externalidades que impulsan a las domésticas a exportar. El estudio para una muestra de 2104 empresas, sugiere que la probabilidad de exportar está positivamente relacionada con la presencia de empresas con capital extranjero, pero no tiene relación alguna con la actividad exportadora en general.

Grether (1999) utiliza datos a nivel de planta para la industria mexicana con el objetivo de verificar el impacto de la presencia extranjera sobre la productividad total de los factores. Los resultados presentados revelan que, aunque existe un 
impacto positivo de las empresas extranjeras sobre la productividad, no existen spillovers sobre las domésticas. Por el contrario, el autor encuentra un impacto negativo de la presencia extranjera sobre la productividad de las plantas domésticas y lo justifica por el hecho de que las trasnacionales se ubican preferentemente en sectores de alta tecnología, lo que conlleva a una menor capacidad de absorción por parte de las empresas domésticas.

Ha habido al menos tres estudios recientes que buscan spillovers de la inversión extranjera en México, los cuales encuentran resultados diversos: Romo (2003), Fujii (2004a) y Brown y Domínguez (2008). Por ello, resulta indispensable revisarlos a detalle.

Romo (2003) busca determinar si la presencia extranjera ha contribuido a crear canales para transmisión de spillovers a empresas domésticas en México. Cuatro mecanismos (efectos de eslabonamiento, colaboración, demostración y entrenamiento) se proponen como canales para spillovers. Los resultados que reporta el estudio son positivos para algunos mecanismos pero negativos para otros. El autor concluye, por lo tanto, que no es posible tener resultados contundentes, pues ello depende del tipo de mecanismo que se utilice para afirmar que existen spillovers en la industria.

Fujii (2004a) presenta un modelo empírico a nivel de empresa con datos recientes sobre productividad y adquisición de tecnología de empresas manufactureras mexicanas (domésticas y con capital extranjero). El modelo empírico que se desarrolla analiza el desempeño de empresas mexicanas (en términos de productividad laboral) en función de la presencia de capitales extranjeros (IED por empresa), de la adquisición de tecnología (del extranjero y del país) y de una serie de variables estructurales. Los resultados encontrados sugieren un impacto negativo de la adquisición de tecnología (en especial, de tecnología proveniente del exterior) sobre la productividad de las empresas mexicanas (tanto domésticas como foráneas).

Brown y Domínguez (2008) plantean un modelo empírico para la industria mexicana a nivel sectorial dentro del periodo 1994-2001, con el objetivo de evaluar la presencia de spillovers de la IED en presencia de capacidades tecnológicas en las empresas nacionales para absorber la tecnología, operarla y mejorarla. El 
FuJII OLEChKo / TORRES GONZÁLEZ La industria mexicana a principios del siglo XXI:

las capacidades tecnológicas y la inversión extranjera como determinantes

resultado del estudio revela que el impacto positivo de la presencia de capital extranjero se observa únicamente en establecimientos nacionales con altas capacidades tecnológicas.

En resumen, no hay resultados concluyentes, tanto en el efecto como en el tipo de sectores respecto al impacto de la inversión extranjera directa sobre la industria mexicana en la forma de spillovers, al menos en los años recientes.

\section{LA IED ASOCIADA A CAPACIDADES TECNOLÓGICAS}

El impacto de la IED sobre una empresa doméstica depende mucho de las características del tipo de sector receptor de tal inversión, dado que es muy relevante verificar que éste sea un sector que ha desarrollado o no capacidades tecnológicas en los años recientes.

Si el sector receptor de la IED no ha desarrollado capacidades tecnológicas, la inversión extranjera llegará en forma de productos (embodied technology) y la empresa no podrá aprender mucho sobre los nuevos procesos de producción. Sin embargo, si el sector pertenece al grupo de industrias que ha desarrollado capacidades tecnológicas, puede ser que la matriz de la empresa trasnacional decida establecer un joint venture con alguna empresa de este sector o que incluso permita que una parte del proceso de producción se lleve a cabo en la empresa doméstica, dado que se va a aprovechar el conocimiento adquirido previamente.

Al menos tres estudios recientes se enfocan a esta discusión, Romo (2005), Pérez-Escatel y Pérez Veyna (2009), y Romo y Hill de Tito (2010). En los tres análisis se parte de una premisa fundamental: la recepción e implementación de los capitales extranjeros, así como de la nueva tecnología, no es de ninguna manera homogénea, sino que depende en gran medida de las capacidades tecnológicas que haya generado la empresa doméstica. Por la importancia que tienen estos estudios para el presente trabajo, a continuación se revisan a detalle.

Romo (2005) encuentra que no sólo es importante saber el nivel de capacidades tecnológicas de los sectores receptores de IED, sino los canales de derrama tecnológica y de comunicación que hay entre empresas nacionales y extranjeras. El autor pretende explorar si el mayor flujo de capital extranjero experimentado 
en la década de1990 por la industria mexicana tiene el potencial de contribuir al desarrollo de capacidades tecnológicas nacionales, así como identificar las políticas que pueden ser aplicadas.

Para los propósitos de su investigación, los canales para la transmisión de derramas se agrupan en cuatro efectos: efectos de eslabonamiento, efectos de colaboración, efectos de demostración y efectos de capacitación. Para las estimaciones se analiza sólo el efecto de eslabonamiento. La hipótesis central es que, los costos asociados con los procesos de subcontratación son una función decreciente de la presencia extranjera en la industria.

El modelo de estimación propuesto por Romo es un Tobit de dos límites para estimar los coeficientes por el método de máxima verosimilitud. La fuente de datos es el ENESTYC 1992 y 1995 para 52 sectores manufactureros a 4 dígitos. Las variables explicativas se agrupan en 3 conjuntos: costos de producción (precios de los factores), una medida de la presencia extranjera en la industria y costos asociados con el efecto de derrama en particular. Para la presencia extranjera se usa: a) el porcentaje de los trabajadores en la industria que son empleados en plantas extranjeras y b) el porcentaje de establecimientos manufactureros en la industria con capital extranjero.

De los resultados del estudio se concluye que no es posible justificar la presencia extranjera en una industria con una relación positiva o negativa con el desarro1lo de los canales para transmitir derramas tecnológicas. La presencia extranjera está relacionada positivamente con efectos de demostración y capacitación, pero la relación se torna negativa para los efectos de colaboración. En los efectos de eslabonamiento no es posible identificar una relación clara.

Posteriormente, Romo hace un análisis de industrias específicas a partir de informes de compañías y entrevistas con empresarios y funcionarios. Las variables dependientes son los mecanismos para transmitir derramas en la industria. Las variables independientes son: a) presencia extranjera; b) dinamismo tecnológico; c) estructura de mercado; d) política gubernamental. Se trabaja con fibras químicas, farmacéutica y electrónica.

Pérez-Escatel y Pérez Veyna (2009) presentan un trabajo cuya hipótesis central plantea que, a medida que la industria va generando capacidades tecnológicas 
FuJII OLEChKo / TORRES GONZÁLEZ La industria mexicana a principios del siglo XXI: las capacidades tecnológicas y la inversión extranjera como determinantes

el país se vuelve más competitivo y que, dichas capacidades están fortalecidas con la presencia de capital extranjero dentro de la industria.

A partir de la Encuesta Industrial Anual y el ENESTYC, y utilizando la metodología de Pavitt (1984) para México, los autores realizan un análisis econométrico de tipo panel para 52 sectores de la industria mexicana en los noventa. El modelo presenta la acumulación de capacidades tecnológicas como variable dependiente, mientras que el número de establecimientos con capital extranjero y el desempeño exportador se consideran como parte de las variables de impacto.

Como resultado principal del análisis los autores encuentran que, la presencia de capital extranjero es significativa en la industria mexicana y tiene un impacto positivo sobre la acumulación de capacidades tecnológicas. Por otro lado, si bien las ventas en el extranjero no resultaron significativas para todos los sectores sí lo son para un grupo de 29 ramas industriales que pertenecen al sector dominado por el proveedor. En este sentido, los autores también encuentran que la acumulación de capacidades tecnológicas es una variable que ayuda a explicar el carácter exportador de las empresas, lo que les permite ser más competitivas internacionalmente.

En el tercero de los trabajos en mención, Romo y Hill de Tito (2010) tienen como objetivo analizar los principales determinantes de la innovación tecnológica en las empresas del sector industrial mexicano. La fuente principal de datos es la Encuesta Nacional de Innovación (ENI) y la metodología está basada en la propuesta de la OCDE (Manual de Oslo). Se presenta un modelo integrado para tener una ecuación que describa cada etapa del proceso de innovación. Esto es, una ecuación para la toma de decisión de innovar o no hacerlo, otra para la determinación de cuánto invertir en actividades de innovación, seguida por una especificación acerca de los productos o resultados de la innovación.

Se proponen cuatro modelos básicos a estimar. Cada uno de ellos cuenta con tres ecuaciones. La primera ecuación modela la decisión de la empresa de comprometerse o no en proyectos de innovación tecnológica; la segunda ecuación mide la cantidad de recursos que se destinan para tal fin (la intensidad de la inversión) $y$, en la tercera ecuación se evalúa el resultado del esfuerzo innovador de distintas maneras: patentes otorgadas a la empresa; porcentaje de ventas totales que se atri- 
buyen a productos innovados; innovación gradual (producto nuevo para la empresa, pero no para el mercado), innovación radical (producto nuevo para la empresa y el mercado); innovación de proceso, producto o proceso y producto.

Los resultados de las estimaciones en la ecuación de "Innova" muestran que el tamaño de empresa está asociado de manera positiva con la probabilidad de innovar. El coeficiente de la cantidad de personas que se dedican a actividades de IyD tiene signo positivo y significativo y de una magnitud considerable. Hay dos resultados que a simple vista parecieran paradójicos: pertenecer a un grupo y contar con capital extranjero presentan un signo negativo. Pero existen varias explicaciones para ello. En el caso de grupo, posiblemente se están formando redes de proyectos de mejoras tecnológicas fuera de la empresa. En cuanto a la IED, puede ser que la transferencia de tecnologías se está haciendo por medio de la importación de maquinaria y equipo que llevan los cambios tecnológicos ya incorporados (embodied technology).

Los resultados de la ecuación de "Inversión" muestran que, el tamaño de la empresa tiene una relación negativa con la cantidad de recursos que se invierten en los proyectos de innovación (como el porcentaje de las ventas totales) pero, la magnitud del impacto es pequeña. La cantidad de trabajadores dedicados a la IyD también está asociada de manera negativa con el monto de la inversión, aunque pierde significancia estadística al introducir la variable de concentración. Nuevamente, el pertenecer a un grupo o contar con IED tiene un impacto negativo en los recursos dedicados a la innovación. El resultado de la IED denota la importancia de distinguir entre tipos de inversión foránea, pues no siempre la presencia de capital extranjero implica el aumento en la innovación de las empresas sino que depende mucho de las capacidades tecnológicas con las que cuentan las compañías y los canales de derrama tecnológica (Romo, 2005).

Las estimaciones de la ecuación de "resultados" muestran que, la inversión que se dedica a los proyectos de innovación está relacionada positiva y significativamente con la obtención de resultados en todas las categorías (proceso; producto; proceso y producto). La magnitud de estos coeficientes disminuye si se considera la concentración de mercado para esa rama de la industria. El tamaño de la empresa tiene escasa relación estadística con el desempeño tecnológico de 
FuJII OlechKo / Torres GonZÁlez La industria mexicana a principios del siglo XXI:

las capacidades tecnológicas y la inversión extranjera como determinantes

las compañías. La IED dentro de la empresa está relacionada con un aumento importante en la probabilidad de innovar en ambos rubros, en producto y en proceso, tanto en magnitud como en relevancia estadística. Este resultado es bastante sólido a la inclusión de la variable de concentración.

Otros estudios recientes sobre IED en México (Dussel, et al., 2003; Dussel, et al., 2007) muestran que, la inversión extranjera no sólo ha impactado en la generación de capacidades tecnológicas, sino que a nivel macroeconómico ha tenido un impacto positivo sobre la tasa de crecimiento por clase industrial generando un mayor dinamismo. Por lo tanto, la IED es una variable que debe ser considerada como un motor de desarrollo de las manufacturas mexicanas.

Dussel, et al. (2003) destacan la IED como una variable a tomar en cuenta cuando se analizan las actividades de ciencia, tecnología e innovación, dado que las características de esta inversión dependen de las particularidades del sector receptor. En este sentido, los autores hacen énfasis en que la difusión tecnológica en el país receptor de IED está directamente relacionada con el diferencial tecnológico respecto a las formas nacionales, siendo favorecidas las economías o sectores altamente tecnificados.

En un trabajo posterior, Dussel et al. (2007) señalan que ha habido escaso análisis sobre las características de los sectores receptores de IED en México, dado que se ha revisado la derrama de la IED sobre la economía en general, pero no se ha planteado la necesidad de externalidades mínimas (generadas por el crecimiento económico) para que pueda ser aprovechado por las empresas con capital extranjero.

Asimismo, dentro de las recomendaciones que hace Dussel et al. (2007) en el trabajo anterior, se señala que es muy importante la modernización de la industria receptora de IED, ya que el capital extranjero será mejor aprovechado por sectores más avanzados en términos de desarrollo de capacidades tecnológicas. Siguiendo esta lógica, los autores encuentran que, a partir de un análisis de panel para las manufacturas mexicanas, además de una serie de variables como el tamaño de mercado, aquellos sectores que han invertido más en IyD tienden a influir en la atracción de mayor IED.

Las capacidades tecnológicas de la industria también pueden verse asociadas a la capacidad de las empresas domésticas de convertirse en proveedoras de las 
multinacionales. En este aspecto, Dussel et al. (2007) encuentran en su estudio empírico a nivel de clase industrial que, la industria mexicana ha sido muy limitada en su papel de abastecedor de insumos para las empresas con capital extranjero, ya que sus elevados costos influyen negativamente en la localización de la IED. Los autores señalan que esta situación ha mermado el impacto que pudiera tener la difusión de las tecnologías externas en el crecimiento económico del país.

Por último, Dussel et al. (2007) encuentran una relación positiva entre gasto en IyD y la IED, lo que implica que las empresas trasnacionales tienden a establecerse en ramas que tienen una mayor inversión y desarrollo tecnológico. Por ello, el autor propone desarrollar la ciencia, tecnología e innovación para atraer más IED al país.

\section{METODOLOGÍA DEL EJERCICIO EMPÍRICO Y BASES DE DATOS EXISTENTES}

En esta sección se planteará la metodología del ejercicio empírico de clasificación de las industrias mexicanas, de acuerdo a las capacidades tecnológicas y a la IED. Primero, expondremos el procedimiento de clasificación High-tech/Low-tech desarrollado en Fujii (2004b; 2010), el cual consiste en separar las industrias que han desarrollado capacidades tecnológicas en años recientes de las que no lo han hecho, de acuerdo al desempeño de un conjunto de variables.

Posteriormente, expondremos las diversas fuentes de información que se encuentran en México para poder realizar este ejercicio con las variables definidas anteriormente. Por último, dado el conjunto de variables representativas de las capacidades tecnológicas, la IED y los contenidos y las características de las fuentes de información, definiremos las variables observadas elegidas para la construcción de una base de datos consistente a utilizarse en el ejercicio empírico.

\section{LA CLASIFICACIÓN HIGH-TECH/LOW-TECH}

La revisión de la literatura realizada en la sección anterior destacó un conjunto de variables asociadas a las capacidades tecnológicas y a la IED. De este conjunto, 
FuJII OlechKo / TORRES GonZÁlez La industria mexicana a principios del siglo XXI: las capacidades tecnológicas y la inversión extranjera como determinantes

el presente trabajo considerará al siguiente grupo de variables para el ejercicio de clasificación industrial:

- Inversiones en investigación y desarrollo (I\&D)

- Capacitación de personal

- Mercados de exportación

- Inversión extranjera directa

La clasificación industrial, de acuerdo a estas variables, se realizará con un ejercicio de separación de industrias en dos grupos, los cuales llamaremos Hightech y Low-tech. Se considera que existe una asociación positiva entre inversión en I\&D, capacitación a personal, orientación hacia el mercado externo y la IED con las capacidades tecnológicas, de tal manera que, entre mayor sea el valor de estas variables es más probable que las industrias desarrollen capacidades tecnológicas. A continuación se enuncian los pasos del ejercicio: ${ }^{5}$

1. Para cada variable $x_{j}$, donde $j=1, \ldots K$ y $K$ es el número de variables, se define un valor crítico $x_{j}^{*}$ que permite dividir a las $i$ industrias en dos grupos, donde $i=1, \ldots, N$ y $N$ es el número de industrias, las cuales guardan relación con un alto o bajo nivel tecnológico.

2. Estas variables, al guardar una relación directa con las capacidades tecnológicas y la inversión extranjera directa, la $i$-ésima industria con un valor en su $j$-ésima variable igual o mayor que el valor crítico correspondiente, $x_{i, j} \geq x_{j}^{*}$, será considerada como High-tech. Si $x_{i, j}<x_{j}^{*}$, entonces será considerada Low-tech.

3. Dado que para cada industria se tendrán $j>1$ variables con una clasificación de High-tech y de Low-tech, la industria en cuestión será, a nivel global, High-tech si la mitad o mayoría de los valores de sus variables son iguales o mayores que el valor crítico, es decir, si las variables en su mayoría son Hightech. En caso contrario serán Low-tech.

\footnotetext{
${ }^{5}$ Las siguientes etapas tienen aparejados distintas posibles particularidades no tratadas en esta sección. Sin embargo, aquellas que se originen del ejercicio empírico realizado en la siguiente sección serán tratadas a detalle.
} 
Panorama ECONÓMICO, vol. VI, núm. 12, enero-junio de 2011

\section{FUENTES DE INFORMACIÓN EXISTENTES Y LIMITANTES DE VARIABLES}

Las características del ejercicio empírico de clasificación industrial dependerán considerablemente de las fuentes de información disponibles. Por tal motivo, es conveniente realizar una búsqueda amplia y un análisis profundo de las fuentes de información que puedan representar a las variables abstractas consideradas arriba.

La principal institución generadora de información económica consultada fue el Instituto Nacional de Estadística y Geografía (INEGI). Esta institución genera información, en diferentes publicaciones, de todas las variables revisadas en el apartado anterior. Sin embargo, para obtener el valor de los flujos de IED en las diferentes industrias es necesario consultar la fuente que genera esta información: la Secretaría de Economía (SE), a través del Registro Nacional de Inversión Extranjera (RNIE), y el Banco de México (BM). Dado que consultamos estas dos últimas fuentes sólo para una variable, comenzaremos con la descripción de la información de la IED.

A partir de 1994 la SE y el BM generan información sobre el valor de la IED con la metodología más reciente, ${ }^{6}$ basada en las recomendaciones sobre balanza de pagos del Fondo Monetario Internacional y en las definiciones de IED de la OCDE (CNIE, 2010). La información que se produce consiste en "[e]l valor de los movimientos de inversión realizados por empresas [ $\left.{ }^{7}\right]$ con IED y notificados al RNIE" (CNIE, 2010:3), por un lado, y en "[u]n monto estimado del valor de los movimientos de inversión de empresas con IED, que se sabe fueron realizados durante el periodo de referencia, pero que aún no han sido notificados al RNIE" (CNIE, 2010:5), por otro lado.

El primero consiste en las notificaciones al RNIE de inversiones realizadas y del valor de importaciones de activo fijo realizadas por empresas maquiladoras

\footnotetext{
${ }^{6}$ Existe información para el periodo 1970-1993 bajo una metodología anterior, la cual no es comparable.

7 Personas físicas o morales extranjeras, sociedades mexicanas con IED en su capital social y empresas fiduciarias participantes en fideicomisos de bienes inmuebles.
} 
FuJII OlechKo / TORRES GONZÁLEZ La industria mexicana a principios del siglo XXI:

las capacidades tecnológicas y la inversión extranjera como determinantes

con inversión extranjera reportado por el BM. Se cuenta con información por tipo de inversión -inversiones iniciales, nuevas inversiones, reinversión de utilidades, cuentas entre compañías, transmisiones de acciones y contraprestación en los fideicomisos de bienes inmuebles-, país de origen de la inversión y sector económico y entidad federativa destino de la inversión.

La información se reporta en dólares corrientes y con una periodicidad trimestral, la cual se produce desde 1994. La clasificación industrial utilizada para el sector económico destino de la inversión es la Clasificación Mexicana de Actividades Productivas (CMAP) y cuenta con una desagregación a nivel de clases económicas. Se puede contar con información, con estas características, de montos anuales de IED por clase de actividad económica bajo el sistema de clasificación CMAP de manera ininterrumpida desde 1994.

El INEGI genera información sobre investigación y desarrollo tecnológico, capacitación a personal, mercados de exportación, entre muchas otras, a través de un conjunto de encuestas realizadas a establecimientos o empresas. El Cuadro 1 sintetiza las principales características de las distintas fuentes de información relacionadas con las variables seleccionadas y para el periodo de tiempo de interés.

Existen cuatro grupos de fuentes de información: 1) Encuesta Industrial Anual (EIA) y Encuesta Industrial Mensual (EIM), 2) Censos Económicos, 3) Encuesta a la Industria Manufacturera, Maquila y Servicios de Exportación (IMMEX) y 4) Encuesta sobre Investigación y Desarrollo Tecnológico (ESIDET). Los primeros dos grupos son parte del trabajo regular de encuestas que realiza el INEGI, mientras que los otros dos las realiza en colaboración con la Secretaría de Economía -en el caso de IMMEX - y con la del Consejo Nacional de Ciencia y Tecnología (CONACYT) -para el de ESIDET-.

En cuanto al periodo de tiempo de disponibilidad de información, aunque su existencia se encuentra en el rango entre 1994 y 2010, existen múltiples discontinuidades que acotarán la temporalidad del estudio empírico. Los Censos Económicos contienen información para un sólo año y se realizan cada cinco. Para el periodo post-2000 contamos con los censos de 2004 y 2009, con información de 2003 y 2008 , respectivamente. 
Panorama EConómico, vol. VI, núm. 12, enero-junio de 2011

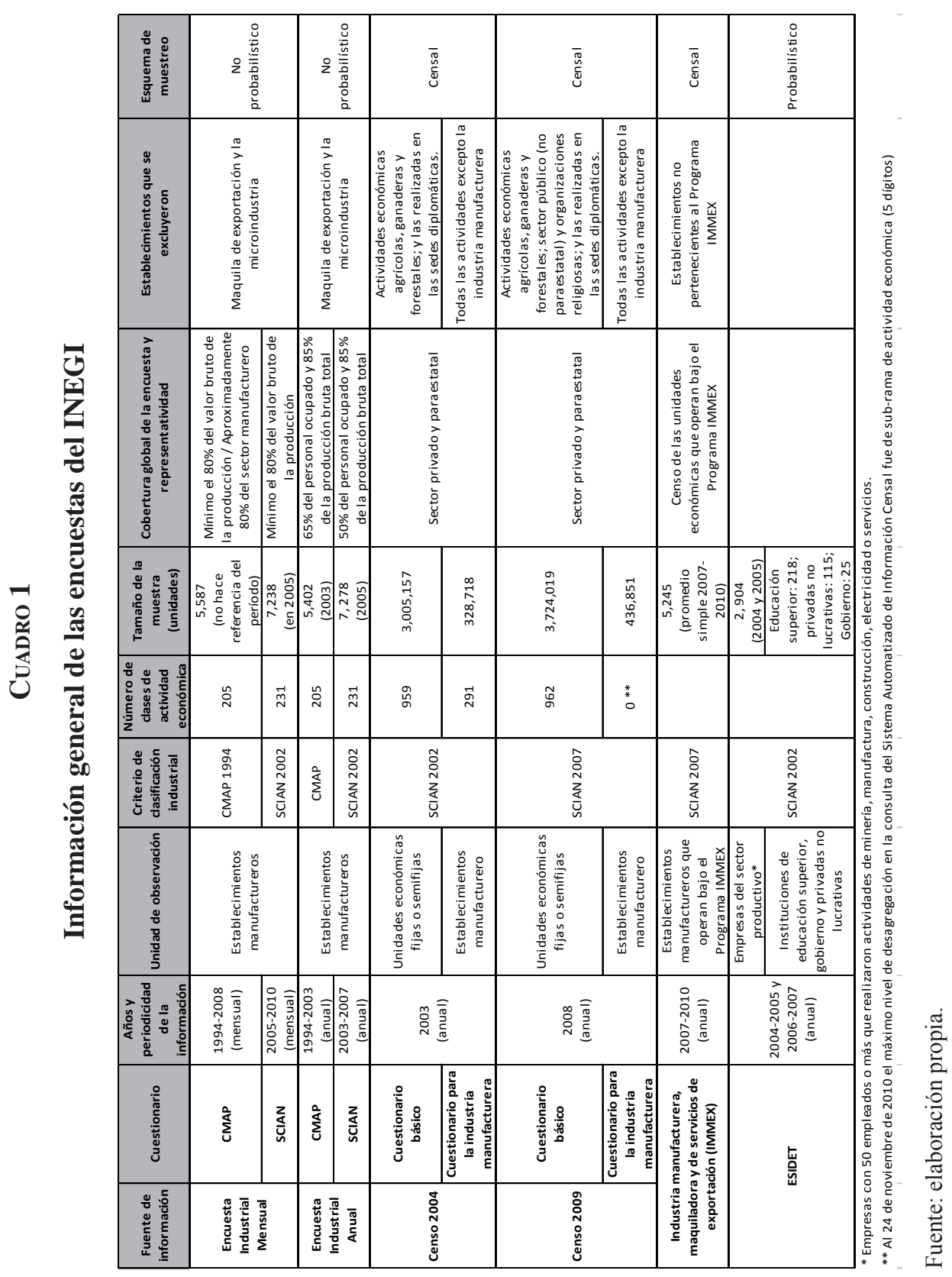


FuJII OLEChKo / TORRES GONZÁLEZ La industria mexicana a principios del siglo XXI:

las capacidades tecnológicas y la inversión extranjera como determinantes

Las encuestas industriales son las que abarcan una mayor periodicidad. ${ }^{8} \mathrm{La}$ EIM-CAMP existe de manera ininterrumpida para el periodo 1994-2008, sin embargo se ha descontinuado para dar paso a la EIM bajo el Sistema de Clasificación Industrial de América del Norte (SCIAN). Para el resto de las encuestas industriales existe una discontinuidad entre 2003 y 2007, por lo que la elaboración de la base de datos con consistencia temporal se puede ver afectada ante la necesidad de tener un periodo anterior y posterior a los años 2003 y 2007. La encuesta del IMMEX y ESIDET se encuentra disponible para periodos de tiempo demasiado cortos (2007-2010 y 2004-2007, respectivamente), por lo que la decisión de su uso se ve complicada si se desea una periodicidad mayor.

Una posible solución a las discontinuidades temporales en la información podría ser la complementariedad entre las encuestas. Sin embargo, como lo muestra la cuarta columna del Cuadro 1, las interrupciones entre las encuestas industriales conviven con un cambio en la clasificación industrial: de la CMAP al SCIAN. Desafortunadamente, para un número considerable de clases económicas, ${ }^{9}$ no es posible realizar la compatibilidad entre clases económicas y con esto construir series de tiempo de mayor duración. ${ }^{10}$ Por tal motivo, la posibilidad de contar con una base para el periodo 1994-2010 sigue estando limitada.

Las encuestas tienen dos unidades de observación distintas: establecimientos y empresas. Todas las encuestas del Cuadro 1 se realizan a nivel de establecimiento, salvo la ESIDET, que se aplica a empresas del sector productivo y a instituciones de educación superior no lucrativas, de gobierno y privadas. Las encuestas industriales se refieren a los establecimientos manufactureros y los censos económicos lo realizan para el total de la economía, con excepción de actividades económicas agrícolas, ganaderas y forestales.

\footnotetext{
${ }^{8}$ La EIA y EIM, bajo el Sistema de Clasificación de América del Norte, se seguirá actualizando en el futuro cercano. Al mes de diciembre de 2010 la información de la EIA tiene como año más reciente al 2007 debido a que se levantó junto con el Censo Económico 2009, del cual en diciembre de 2010 publicaron los resultados definitivos.

9 Incluso a un nivel de agregación de 2, 4 y 5 dígitos.

${ }^{10}$ Véase INEGI (1994).
} 
El nivel máximo de desagregación, tanto del CMAP como del SCIAN, es a 6 dígitos. ${ }^{11}$ Para la industria manufacturera, nuestro gran sector de interés, el número de clases económicas para las encuestas bajo la CMAP son 205 clases mientras que para el SCIAN son 235. El catálogo completo de clases económicas tanto para el CMAP como para el SCIAN es mayor a 205 y 235 clases, respectivamente. Esto es porque, a diferencia de los censos económicos, cuyo objetivo es tratar de incluir todas las actividades económicas, la EIA y la EIM pretenden tener sólo una muestra representativa de la industria manufacturera. ${ }^{12}$

Como lo muestra las columnas 7 y 8 , mientras que los censos encuestan más de 3 millones de establecimientos y tienen una representación del sector privado y paraestatal, la EIA y la EIM encuestan entre 5402 y 7278 establecimientos, los cuales representan entre el 80 y $85 \%$ del valor bruto de la producción manufacturera y entre el 50 y $65 \%$ del personal ocupado. Dado que la información de los establecimientos maquiladores para exportación se encuentra en la encuesta del IMMEX, las EIA y la EIM excluyen a estos establecimientos, además de toda la microindustria. ${ }^{13}$

Con la evaluación de las características generales de las fuentes del INEGI tendríamos la primera parte de la construcción de la base de datos: la fuente de información de origen. La otra parte depende de las variables específicas que queremos incluir en la base y su disponibilidad con las fuentes de información. Dado que ya realizamos este ejercicio para los montos de IED podemos adelantar alguna conclusión sobre las fuentes de información posibles: si el ejercicio empírico incluirá en todas sus variantes los montos de IED significa que 1) sólo podremos tomar fuentes de información cuya clasificación industrial se base en la CMAP y 2) se podrá aprovechar la información de todos los años de las fuentes dado que se cuenta con datos para el rango de tiempo entre 1994-2009. Sin embargo, si en

${ }^{11} \mathrm{Al}$ mes de noviembre, el nivel máximo de desagregación en la información en línea de los Censos Económicos se encontraba a 5 dígitos, es decir, subrama.

12 INEGI (2005a, 2005b) especifica las características de las clases para ser incluidas.

${ }^{13}$ El esquema de muestreo de la EIA y EIM es no probabilístico y los censos económicos y la IMMEX, por construcción es censal. La ESIDET es la única que tiene diseño probabilístico. 
FuJII OlechKo / TORRes GonZÁLeZ La industria mexicana a principios del siglo XXI: las capacidades tecnológicas y la inversión extranjera como determinantes

lugar de montos de IED se puede tomar otro indicador de esta variable, las posibilidades de conformación de la base de datos y sus características cambiarán. A continuación veremos este caso y el del resto de las variables.

El Cuadro 2 contiene un listado de las variables encontradas en las fuentes de información que podrían representar las variables abstractas de I\&D, capacitación a personal, mercado exterior y la IED, además de señalar la ubicación específica en la fuente de información. De inicio, este análisis se realizó sin la participación de la ESIDET y la IMMEX debido a que, además de su corta periodicidad, la información de la IMMEX en internet no se encuentra a nivel de industrias sino de entidades federativas, y por otro lado no existe disponibilidad de información en línea ni en formato impreso de la ESIDET; se tiene que obtener por medio de una solicitud al INEGI o al CONACYT.

Dentro de las variables asociadas a la I\&D, la que encontramos más relevante fue el gasto en investigación y desarrollo tecnológico (GI\&DT), cuya disponibilidad se encuentra en la EIA-CMAP y en los Censos Económicos 2004. Dentro del cuestionario de manufacturas del Censo Económico 2004 existe una sección dedicada a innovación e investigación. Integramos el GI\&DT debido a que deseamos incluir una medida monetaria del esfuerzo en esta actividad y a que el resto de las preguntas eran de respuesta binaria (si/no). Esta variable se encuentra dividida en dos conceptos: el GI\&DT en el proceso productivo y el GI\&DT en la prevención y control de la contaminación. Esta información no se encuentra disponible en el sitio web del INEGI y para obtenerla se tuvo que realizar una solicitud.

Desafortunadamente, esta variable dejó de incluirse en los cuestionarios de las encuestas industriales basadas en el SCIAN y del Censo Económico 2009. De acuerdo a la comunicación electrónica con personal del INEGI, la ausencia de esta información en las siguientes encuestas se debe a la poca respuesta que han obtenido en los establecimientos. ${ }^{14}$ Esta es una de las razones por las que esta información no se publica como la mayoría de las variables de los cuestionarios. La razón es que esta información es controlada por la empresa y en menor medida por los establecimien-

\footnotetext{
${ }^{14}$ En sus palabras, no cuentan con la "consistencia y confiabilidad requeridas".
} 
PANorama EConómico, vol. VI, núm. 12, enero-junio de 2011

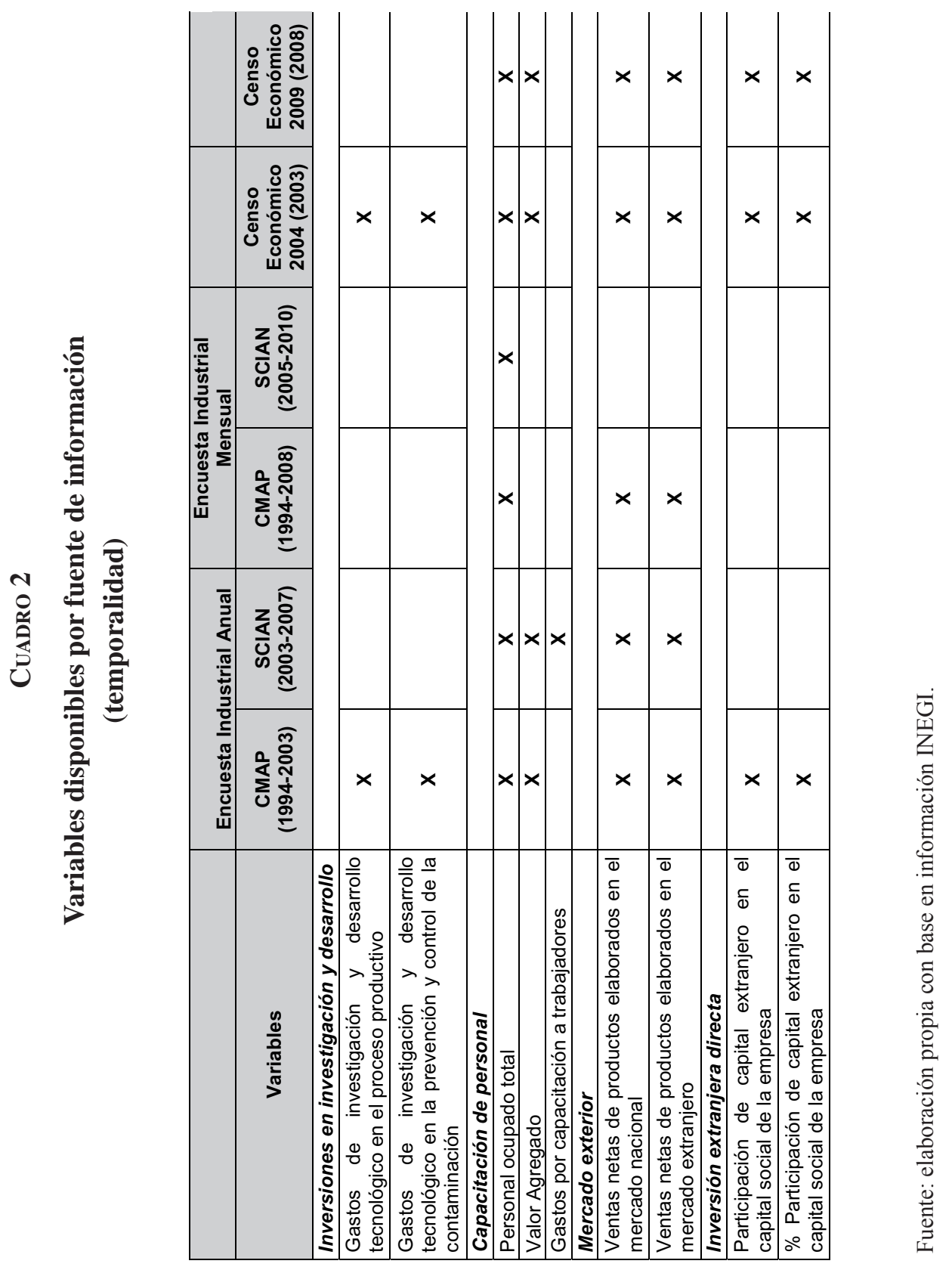


FuJII OLEChKo / TORRES GONZÁLEZ La industria mexicana a principios del siglo XXI: las capacidades tecnológicas y la inversión extranjera como determinantes

tos. Incluso, se nos comunicó que para el año 2003 la respuesta fue tan limitada que la información disponible por solicitud de esta variables es hasta 2002.

Ante estas características, la inclusión de esta variable limitará el periodo de estudio a 1994-2002 y tendrá que utilizarse la CMAP para el periodo 1994-2002 (EIA) o el SCIAN para 2003 (Censos Económicos).

Respecto a la capacitación de personal, pudimos identificar dos posibles variables: productividad y gasto por capacitación a los trabajadores. La productividad, definida como el valor agregado por personal ocupado total, se puede obtener de todas las fuentes del Cuadro 2, con excepción de las encuestas mensuales. Sin embargo, el gasto por capacitación a trabajadores sólo se encuentra en la EIASCIAN. Además, esta información no se encuentra desglosada en la información del sitio web del INEGI, ya que viene agregada dentro de "otros gastos".

Para la orientación a los mercados externos, utilizaremos una medida de la participación del valor de las exportaciones sobre el valor total. Dados los conceptos y su desglose encontrados en las encuestas, se decidió utilizar el cociente entre el valor de las ventas netas de mercancías al mercado externo y el valor de las ventas netas totales - mercado externo más mercado nacional-. Salvo para la EIM-SCIAN, la construcción de esta variable es posible para todas las fuentes de información.

Por último, la información de las encuestas del INEGI permite tener otros indicadores de la IED, además del valor de ésta proporcionado por la SE y el BM. La EIA-CMAP y los Censos Económicos 2004 y 2009 preguntan tanto de la existencia o no de IED en el capital social de la empresa como de su porcentaje. Esta información comprende la de grandes empresas -i.e., excluye PYMES-, sin incluir la refinación de petróleo. No obstante, no se encuentra disponible en el sitio web del INEGI y tiene que solicitarse como tabulado especial..$^{15}$

\footnotetext{
15 Adicionalmente, por cuestiones de confidencialidad de información, el nivel de desagregación se puede ver limitado. Dussel (2009) menciona que sólo fue posible obtener información a nivel de rama -4 dígitos-.
} 
Panorama ECONÓMICO, vol. VI, núm. 12, enero-junio de 2011

\section{VARIABLES INCLUIDAS Y SUS CARACTERÍSTICAS EN LA CLASIFICACIÓN INDUSTRIAL HIGH-TECH/LOW-TECH}

Considerando las fuentes de información disponibles, sus características y las variables candidatas, la base de datos que construimos para el ejercicio estadístico está compuesta por las siguientes variables y sus características:

1. Variables:

a. Valor en dólares corrientes de los flujos de IED a México.

b. Valor en pesos corrientes del GI\&DT en el Proceso Productivo (GI\&DTPP), el GI\&DT en la Prevención y Control de la Contaminación (GI\&DTPCC) y GI\&DT Total $(G I \& D T T=G I \& D T P P+G I \& D T P C C)$. Todas las variables estarán ponderadas por el valor de la Producción Bruta Total $(P B T)$.

c. Productividad $(P)$, definida como el Valor Agregado Bruto Total (VABT) en pesos corrientes dividido por el Personal Ocupado Total (POT).

d. Participación porcentual del valor en pesos corrientes de las Ventas Netas al Mercado Extranjero $(V N X)$ respecto del valor en pesos corrientes de las Ventas Netas Totales $(V N T)$, compuestas por las VNME y las Ventas Netas al Mercado Nacional ( $V N M N)$.

2. Fuentes de información: SE y BM para la IED y la EIA del INEGI.

3. Periodo de estudio: 1994-2002. Dado que la información del GI\&DT llega hasta 2002, no pudimos aprovechar la disponibilidad del resto de las variables a 2003.

4. Sistema de clasificación industrial y nivel de agregación: CMAP, clases económicas (6 dígitos).

5. Número de industrias: 204. La información de la EIA-CMAP contiene las clases "Elaboración de malta" y "Fabricación de cerveza", con códigos 313040 y 313041, respectivamente, mientras que la base de datos de IED contiene la clase económica "Industria de la Cerveza y de la Malta", con código 313040, y no incluye la 313041, que en la EIA es "Fabricación de cerveza". Ante la aparente agregación de elaboración de malta y fabricación de cerveza en la clase 313040 por parte de los montos de IED, hemos decidido agregar las clases $313040 \mathrm{y}$ 313041 de la EIA, con lo que se redujo una clase de actividad económica. 
FuJII OlechKo / TORRes GonZÁLeZ La industria mexicana a principios del siglo XXI: las capacidades tecnológicas y la inversión extranjera como determinantes

\section{CLASIFICACIÓN DE LA INDUSTRIA MEXICANA EN 1994-2002}

Con las seis variables elegidas realizaremos dos ejercicios de clasificación industrial: uno utilizando tres variables y otro utilizando cinco variables, de tal manera que $K=3$ y $K=5$, respectivamente. Para $K=3$ las variables serán $G I \& D T T / P B T$, $P$ e $I E D$, mientras que para $K=5$ serán GI\&DTPP/PBT, GIDTPCC/BPT, $P, I E D$ y $V N X / V N T$.

Definidas las variables para cada ejercicio, es necesario especificar el valor crítico $x_{j}^{*}$ que separará las industrias en High-tech y Low-tech. Contamos con datos tipo panel, es decir, $i=1, \ldots, 204$ individuos para los cuales existen $t=1, \ldots, 9$ periodos de tiempo. Para el presente ejercicio se elegirá $x_{j}^{*}=(1 / N) \sum_{i=1}^{N} \bar{x}_{j}$, donde $\bar{x}_{i j}=(1 / T) \sum_{t=1}^{T} x_{i t j}$, es decir, para cada variable elegiremos el promedio industrial de los promedios temporales de cada industria.

El Cuadro 3 nos muestra los resultados globales de las industrias de clasificación High-tech, H, y Low-tech, L, considerando que si $x_{j} \geq x_{j}^{*}$ ó $x_{j}<x_{j}^{*}$. Para el ejercicio con tres variables, el 19.6\% de las industrias resultaron High-tech de acuerdo al los criterios establecidos. De éstas, 12 clases de actividad económica tuvieron en todas sus variables la calificación Hig-tech, HH. Estas industrias son las de harina de maíz, cigarros, cartón, libros, resinas, farmacéuticos, pinturas, jabones, productos químicos secundarios, aceites lubricantes, aparatos de comunicación y automóviles y camiones. El número de industrias con dos variables Hightech, $2 H$, es poco más del doble. Del $80.4 \%$ de industrias Low-tech, 75 tienen dos variables Low-tech, $2 L$, mientras que 89 son todas Low-tech, $L L$.

En cuanto al ejercicio con cinco variables, se incrementó el número de clases económicas clasificadas como High-tech al pasar de 38 a 49, representando ahora el $24 \%$ del total. Este movimiento se dio con la disminución de una industria con desempeños en todas sus variables por encima de la media: productos químicos secundarios, la cual también se encuentra en las $H H$ del ejercicio de tres variables. Las clases con tres o cuatro variables High-tech, $3 \mathrm{H}$ y $4 \mathrm{H}$, respectivamente, constituyen 48 de las 49 industrias High-tech. De las industrias Low-tech, poco más de un tercio fueron las que tuvieron desempeños por debajo de la media en todas las variables. 
Por último, en el paso del ejercicio de tres a cinco variables, 36 clases económicas fueron High-tech en ambos ejercicios, 13 pasaron de Low-tech a High-tech y 4 dejaron de ser High-tech.

\section{CONCLUSIONES Y RECOMENDACIONES DE POLÍTICAS}

El documento ha destacado el papel fundamental de las capacidades tecnológicas como determinantes de la industria manufacturera en México, sobre todo durante el periodo de apertura iniciado en la década de los 80. Asimismo, se destacó la importancia de la IED en la adquisición y desarrollo de dichas capacidades tecnológicas.

Dentro de las variables que podrían fomentar la adquisición y desarrollo de las capacidades tecnológicas se enfatizó la IED, las actividades de investigación y desarrollo, la capacitación laboral y la orientación hacia los mercados externos. La búsqueda de variables observadas en la industria mexicana de principios del siglo XX que representen a estas variables abstractas, nos llevó a realizar un análisis profundo de las fuentes de información, y sus características, que publican diversas instituciones. Pudimos apreciar que los cambios ocurridos en la adopción de sistemas de clasificación de actividades económicas a mediados de la primera década del siglo XX, limita drásticamente la posibilidad de realizar ejercicios empíricos sobre la industria manufacturera. Asimismo, señalamos la ausencia de información de montos de IED bajo el sistema de clasificación SCIAN.

Los ejercicios de clasificación industrial por nivel tecnológico, High-tech y Low-tech, ubicaron entre 38 y 45 clases económicas - de 204 consideradas- como industrias High-tech, lo cual representa alrededor del 20\% del total. La realización del ejercicio nos permitió observar bajo qué variables se consideran High-tech

y Low-tech. Fueron pocos los casos en que todas las variables de las industrias resultaron ser High-tech. En la mayoría de los casos existen combinaciones entre indicadores de High-tech con Low-tech.

A diferencia del ejercicio de Fujii (2010), al incorporar la variable IED para esta clasificación la proporción de sectores que han generado capacidades tecno- 
FuJII OlechKo / TORRes GONZÁLEZ La industria mexicana a principios del siglo XXI: las capacidades tecnológicas y la inversión extranjera como determinantes

lógicas en años recientes (catalogadas como High-tech) disminuye un poco (en Fujii 2010, la proporción de High-tech fue del 27\%). Esto puede atribuirse al tiempo que necesitan las empresas receptoras de capital extranjero para asimilar las tecnologías avanzadas y para mantener el ritmo de inversión en actividades de investigación y desarrollo.

La clasificación presentada en este trabajo permite identificar los factores detonantes de capacidades tecnológicas, representadas por las variables consideradas para el estudio, que se encuentran rezagadas en desempeño para elaborar políticas públicas encaminadas a su desarrollo. Esto debe de ser especialmente relevante para las políticas de desarrollo tecnológico e innovación, tanto de carácter federal como estatal y municipal.

\section{BIBLIOGRAFÍA}

Aitken, B. y A. Harrison (1991), Are There Spillovers from Foreign Direct Investment? Evidence from Panel Data for Venezuela, mimeo, Universidad de Columbia.

Aitken, B. G. Hanson y A. Harrison (1997), "Spillovers, Foreign Investment and Export Behavior”, Journal of International Economics, num. 43, pp. 103-132.

Aitken, B. y A. Harrison (1999), "Do domestic firms benefit from Direct Foreign Investment? Evidence from Venezuela", American Economic Review, Vol. 89, num. 3, pp. 605-619.

Arias, A. (2003), "Mecanismos de aprendizaje y capacidades tecnológicas: el caso de una empresa del sector curtidor", en Aboites J. y G. Dutrenit (eds.) Innovación, aprendizaje y creación de capacidades tecnológicas, UAM-X, M.A. Porrúa, México.

Benavente, J. (2006), "The role of research and innovation in promoting productivity in Chile", Economics of Innovation and New Technology, Vol. 15, num. 4/5, pp. 301-315.

Blomström, M. (1986), "Multinationals and Market Structure in Mexico", World Development, Vol. XIV (4), 523-530. 
Panorama ECONÓMICO, vol. VI, núm. 12, enero-junio de 2011

Blomström. M. y A. Kokko (1997), "How Foreign Investment Affects Host Countries", Policy Research Working Paper, 1745, The World Bank International Economics Department.

Blomström, M., A. Kokko y M. Zejan (1993), "Host Country Competition, Labour Skills, and Technology Transfer by Multinationals", NBER, Working Paper, 4131.

Blomström, M. A. Kokko y M. Zejan (2000), Foreign Direct Investment: Firm and Host Country Strategies, Macmillan, Londres.

Blomström, M. y A. Persson (1983), "Foreign Investment and Spillover Efficiency in an Underdeveloped Economy: evidencie from the Mexican manufacturing Industry", World Development, Vol. 11, num. 6, pp. 493-501.

Blomström, M. y F. Sjohölm (1999), “Technology Transfer and Spillovers: Does Local Participation with Multinationals Matter?", European Economic Review, num. 43.

Chudnovsky, D., A. López y G. Pupato (2004), "Innovation and productivity in developing countries: A study of Argentine manufacturing firms' behavior (1992-2001)", Research Policy, Vol. 35, pp. 266-288.

Cohen, W.M. y D.A. Levinthal (1990), “Absorptive capacity: a new perspective on learning and innovation", Administrative Sciences Quarterly, Vol. XXXV (1), 128-152.

Comisión Nacional de Inversiones Extranjeras (CNIE) (2010), Informe estadístico sobre el comportamiento de la Inversión Extranjera en México, EneroSeptiembre, México, D.F.

Dosi, G., C. Freeman, R. Nelson y L. Soete (1988), Technical Change and Economic Theory, Pinter, London, U.K.

Duguet, E. (2006), "Innovation height, spillovers and TFP growth at a firm level: evidence from French manufacturing", Economics of Innovation and New Technology, Vol. 15, num. 4/5, pp. 415-442.

Dunning, J. (1977), "Trade, Location of Economic Activity and the MNE: a search for an Eclectic Approach", en B. Ohlin, P. Hesselborn y P. Wijkman (eds.), The International Allocation of Economic Activity, Mcmillian, Londres. 
FuJII OlechKo / TORRes GONZÁLEZ La industria mexicana a principios del siglo XXI: las capacidades tecnológicas y la inversión extranjera como determinantes

Dussel, E. (2009), Don't Expect Apples From a Pear Tree: Foreign Direct Investment and Innovation in Mexico, Discussion Paper Number 28, Working Group on Development and Environment in the Americas.

Dussel, E. (coord.), L. M. Galindo y E., Loría (2003), Condiciones y efectos de la inversión extranjera directa y del proceso de integración regional en México durante los noventa, Facultad de Economía UNAM, BID y Plaza y Valdés, México.

Dussel, E. (coord.), L. M. Galindo, E. Loría y M. Mortimore (2007), Inversión Extranjera Directa en México: Desempeño y Potencial, Facultad de Economía UNAM, Cechimex, Secretaría de Economía y Siglo XXI, México.

Eeckhout, J. y B. Jovanovic (2002), "Knowledge spillovers and inequality", American Economic Review, Vol. 95, num. 5, pp. 1290-1307.

Fransman, M. (1985), "Technological capability in the Third World: an overview and introduction”, en M. Fransman y K. King, Technological capability in the Third World, Macmillan, Londres.

Fransman, F. y K. King (1985), Technological Capability in the Third World, Macmillian, London, U.K.

Fujii, D. (2004a), "Inversión Extranjera y Productividad en México", Investigación Económica, Vol. LXIII, núm. 248, pp. 147-173.

Fujii, D. (2004b), "La tecnología y el éxito industrial en México: una propuesta de división sectorial", Revista de Economía Mundial, 10/11, pp. 105-126.

Fujii, D. (2010), Capacidades tecnológicas e innovación en la industria manufacturera del Estado de México, El Colegio Mexiquense, A.C. y Consejo Mexiquense de Ciencia y Tecnología.

Globerman, S. (1979), 'Foreign Direct Investment and 'Spillover' Efficiency Benefits in Canadian Manufacturing Industries", Canadian Journal of Economics, Vol. 12.

Grether, J. (1999), "Determinants of Technological Diffusion in Mexican Manufacturing: a Plant-Level Analysis", World Development, Vol. 27, num. 7, pp. 1287-1298.

Hadad, M. y A. Harrison (1993), "Are there positive Spillovers from Direct Foreign Investment?”, Journal of Development Economics, num. 42, pp. 51-74. 
Hernández, C. A. y L. M. Sánchez (2003), “Aprendizaje tecnológico y dinámica industrial”, en Aboites, J. y G. Dutrenit (eds.) Innovación, aprendizaje y creación de capacidades tecnológicas, UAM-X, M.A. Porrúa, México.

Instituto Nacional de Estadística y Geografía (INEGI) (1994), Tablas comparativas SCIAN México 2002-CMAP 1994, recuperado del sitio <http://www. inegi.org.mx/sistemas/scian/contenidos/Tablas\%20comparativas/Tabla\%20 Comparativa\%20VI.pdf $>$.

Instituto Nacional de Estadística y Geografía (INEGI) (2005a), Síntesis Metodológica de la Encuesta Industria Anual, Aguascalientes.

Instituto Nacional de Estadística y Geografía (INEGI) (2005b), Síntesis Metodológica de la Encuesta Industrial Anual, edición especial, de 231 clases de actividad económica, Aguascalientes.

Kokko, A. (1994), “Technology, Market Characteristics and Spillovers”, Journal of Development Economics, num. 43, pp. 279-293.

Kokko, A., R. Tansini y M. Zejan (1996), "Local Technological Capability and Spillovers from FDI in the Uruguayan Manufacturing Sector", Journal of Development Studies, Vol. 34, pp. 602-611.

Krugman, P. y M. Obstfeld (2000), International Economics: Theory and Policy, Addison-Wesley, Estados Unidos.

Lall, S. (1992), “Technological capabilities and industrialization”, World Development, Vol. XX, num. 2, pp. 165-186.

Lööf, H. y A. Heshmati (2006), “On the relationship between innovation and performance: a sensitivity analysis", Economics of Innovation and New Technology, Vol. 15, num. 4/5, pp. 317-344.

Moran, T. (1998), Foreign Direct Investment and Development, Washington Institute for International Economics.

Markusen, J. (1996), "Multinational Firms, Location and Trade", The Worls Economy, Vol. 21, num. 6, pp. 733-757.

Nelson, R. y S. Winter (1982), An Evolutionary Theory of Economic Change, Harvard University Press, Cambridge, Mass.

Pavcnik, N. (2002), "Trade liberalization, exit and productivity improvement: evidence from Chilean palnts", Review of Economic Studies, Vol. 69, num. 1, pp. 245-276. 
FuJII OlechKo / TORRes GONZÁLEZ La industria mexicana a principios del siglo XXI: las capacidades tecnológicas y la inversión extranjera como determinantes

Pérez-Escatel, A. y O. Pérez Veyna (2009), Competitividad y acumulación de capacidades tecnológicas en la industria manufacturera mexicana, Investigación Económica, Vol. LXVIII, núm. 268, pp. 159-187.

Romo, D. (2003), "Derramas tecnológicas de la inversión extranjera en la industria mexicana", Comercio Exterior, Vol. 53, núm. 3, pp. 230-243.

Romo, D. (2005), Inversión Extranjera, Derramas Tecnológicas y Desarrollo Industrial en México, FCE, México, D.F.

Romo, D. y P. Hill de Tito (2010), "Los determinantes de las actividades tecnológicas en México" en Bazdresch C. y L. Meza (eds.), La tecnología y la innovación como motores del crecimiento en México, FCE, México, D.F., pp. 73-135.

Schumpeter, J. (1934), The Theory of Economic Development: An inquiry into profits, capital, credit, interest and the business cycle, Harvard University Press.

Sjöholm, F. (1999), “Technology Gap, Competition and Spillovers from Direct Foreign Investment: Evidence from Establishment Data", The Journal of Development Studies, num. 36(1), pp. 53-73.

Stern, R. (1973), The Balance of Payments: Theory and Economic Policy, Mcmillan, Londres.

Stoneman, P. (ed.) (1995), Handbook of the economics of innovation and technological change, Blackwell.

Teece, D., R. Rumelt, G. Dosi y S. Winter (1994), "Understanding Corporate Coherence", Journal of Economic Behaviour and Organization, num. 23, pp. $1-30$. 